\title{
Co-inhibition of miRNA-21 and miRNA-221 induces apoptosis by enhancing the p53-mediated expression of pro-apoptotic miRNAs in laryngeal squamous cell carcinoma
}

\author{
XUAN KAN, YANAN SUN, JIANGUANG LU, MINGHUA LI, YU WANG, \\ QIUYING LI, YING LIU, MING LIU and LINLI TIAN
}

Department of Otolaryngology, Head and Neck Surgery, The Second Affiliated Hospital, Harbin Medical University, Harbin, Heilongjiang 150081, P.R. China

Received March 18, 2015; Accepted March 3, 2016

DOI: $10.3892 / \mathrm{mmr} .2016 .5048$

\begin{abstract}
Dysregulation of a numerous microRNAs (miRNAs) has been implicated in laryngeal squamous cell carcinoma (LSCC). Among those miRNAs, miR-21 and miR-221 are co-overexpressed and commonly target the phosphatase and tensin homolog protein (PTEN) that is located in the PTEN-Akt signaling pathway. The present study investigated whether co-inhibition of miR-21 and miR-221 induced synergistic apoptosis of human LSCC cells. Methyl thiazolyl tetrazolium (MTT) and terminal deoxynucleotidyl-transferase-mediated deoxynucleotide triphosphate nick end labeling (TUNEL) assays were used to observe the potential effect of miR-21 and miR-221 on cell viability and apoptosis in cells co-transfected with anti-miRNA oligonucleotide (AMO)-21 and AMO-221. The protein expression levels of PTEN, Akt and p53 were determined by western blotting. The cellular abundance of 6 pro-apoptotic miRNAs transcribed by p53 mediation, consisting of miR-15a, miR-16-1, miR-26a, miR-34a, miR-143 and miR-203, was measured with using reverse transcription-quantitative polymerase chain reaction (RT-qPCR). MTT results indicate that in vitro co-transfection of AMO-21 and AMO-221 leads to a decline in cell viability, compared with the transfection of AMO alone. This result was verified by the detection of apoptosis using TUNEL assays. Co-transfection of AMO-21 and AMO-221 resulted in a marked reduction in Akt phosphorylation and enhanced expression of PTEN and p53 were observed; consequently, leading to an amplification of the transcription of 6 pro-apoptotic miRNAs.
\end{abstract}

Correspondence to: Professor Ming Liu or Professor Linli Tian, Department of Otolaryngology, Head and Neck Surgery, The Second Affiliated Hospital, Harbin Medical University, 246 Xuefu Road, Harbin, Heilongjiang 150081, P.R. China

E-mail: liumingorl@163.com

E-mail: tianlinli0rl@163.com

Key words: laryngeal squamous cell carcinoma, synergistic, microRNA-21, microRNA-221, apoptosis
The present findings confirmed that co-inhibition of miR-21 and miR-221 synergistically triggers cell apoptosis in vitro. The altered PTEN-Akt signaling and p53-mediated amplification of the transcription of pro-apoptotic miRNAs may be involved in the observed synergistic effect. The present study provides novel insights into the mechanism underlying apoptosis-associated miRNA-miRNA mutual regulation in LSCC.

\section{Introduction}

Laryngeal squamous cell carcinoma (LSCC) is one of the most common head and neck cancers in the world and accounts for almost $90 \%$ of all malignant laryngeal cancers (1). LSCC is accompanied by progressively impaired laryngeal function that seriously affects the quality of life of those affected by the disease $(2,3)$. Currently, the primary clinical interventions for LSCC are invasive radiotherapy and surgery. Surgical procedures, such as a total laryngectomy, are common, particularly in patients with advanced LSCC (4). Over the previous decades, novel therapeutic strategies have been developed and introduced for the clinical treatment of LSCC; however, no significant improvement in the prognosis of this disease has been achieved (5). Thus, an understanding of the molecular mechanisms underlying the pathology of LSCC is crucial for the identification of novel therapeutic targets for optimizing treatment.

Previous studies have suggested that there are numerous microRNAs (miRNAs) with aberrant expression in LSCC tissue, which may be potential therapeutic targets $(6,7)$. miRNAs are endogenous, single-stranded, non-coding RNAs, $\sim 20$ nucleotides long. Of the numerous LSCC-associated miRNAs that were identified by miRNA microarray analysis, miR-21 was subsequently found to be upregulated (7). In addition, miR-21 was shown to function as a potent LSCC tumor promoter by inhibiting cell apoptosis through the regulation of Ras expression (8). miR-221 was confirmed in vitro to induce the proliferation of laryngeal cancer cells via targeting apoptotic protease activating factor-1 (Apaf-1) in in vitro studies (9). Bioinformatics analysis indicated that the overexpression of the LSCC-associated miRNA miR-221 may synergistically act 
with miR-21 in cell apoptosis (10). Additionally, experimental evidence revealed that the phosphatase and tensin homolog (PTEN) gene was associated with apoptosis in LSCC (11) and may be targeted by miR-21 and miR-221 $(12,13)$. This finding was consistent with the observation of low expression of the PTEN gene and protein in a HEp-2 LSCC cell line $(11,14)$. PTEN is an important negative regulatory factor of Akt (15), and the activation of PTEN is associated with LSCC metastasis (16). As a result, Akt negatively controls the expression level of the tumor-suppressor gene tumor protein p53 (TP53) in cancer cells (17). Low levels of p53, which is the protein product of the TP53 gene, aid the growth of LSCC tumors, as p53 is a common transcriptional activator of numerous pro-apoptotic miRNAs, including miR-15a, miR-16-1, miR-26a, miR-34a, miR-143 and miR-203 (18).

Overall, the accumulated data indicate that simultaneous overexpression of miR-21 and miR-221 in LSCC may aggregately weaken the negative regulation of Akt by PTEN, resulting in the inefficiency of p53 to activate the transcription of pro-apoptotic miRNAs. Based on this hypothesis, the present study considered that co-inhibition of miR-21 and miR-221 may contribute to a reversal of the imbalance between anti-apoptotic and pro-apoptotic miRNAs established in LSCC. To investigate the validity of the present hypothesis, an in vitro experiment was performed, in which anti-miRNA oligonucleotide (AMO)-21 and AMO-221 were co-transfected into the LSCC Hep-2 cell line. Cellular apoptosis and the protein expression levels of PTEN and phosphorylated-Akt (p-Akt) were monitored. The changes in the cellular abundance of the aforementioned 6 pro-apoptotic miRNAs were also investigated. The aim of the present study was to offer a potential miRNA-based therapeutic method for the treatment of LSCC.

\section{Materials and methods}

Cell culture and miRNA transfection. The LSCC Hep-2 cell line was purchased from the Institute of Biochemistry and Cell Biology, Chinese Academy of Science (Shanghai, China). The cells were maintained in Gibco Dulbecco's modified Eagle's medium (Thermo Fisher Scientific, Inc., Waltham, MA, USA) supplemented with $10 \%$ Gibco fetal bovine serum (FBS; Thermo Fisher Scientific, Inc.), 100 $\mu \mathrm{g} / \mathrm{ml}$ penicillin (Beijing Leagene Biotech Co., Ltd., Beijing, China) and $100 \mu \mathrm{g} / \mathrm{ml}$ streptomycin (Beijing Leagene Biotech Co., Ltd.). Subsequently, the cells were transferred to 96-well plates and cultured in fresh medium, without antibiotics. For transfection, cells were washed with serum-free medium once and then incubated with $4 \mathrm{ml}$ serum-free medium for 4-6 $\mathrm{h}$. AMO-21 or AMO-221 and Invitrogen Lipofectamine 2000 (Thermo Fisher Scientific, Inc.) were separately mixed with $500 \mu 1$ Gibco Opti-MEM I Reduced Serum medium (Thermo Fisher Scientific, Inc.) for $5 \mathrm{~min}$. Then, the two mixtures were combined and incubated for $20 \mathrm{~min}$ at room temperature. The Lipofectamine 2000 and AMO-21 or AMO-221 mixture was added to the cells and incubated at $37^{\circ} \mathrm{C}$ for $6 \mathrm{~h}$. Subsequently, $5 \mathrm{ml}$ fresh medium containing 10\% FBS was added to the flasks, and the cells were maintained in the culture conditions until use.

Methyl thiazolyl tetrazolium (MTT) assays. The colorimetric MTT assay was used to assess the viability of cells cultured in 96-well culture plates. The dye measures mitochondrial dehydrogenase activity and utilizes the fact that viable cells, rather than dead cells, reduce MTT. Subsequent to AMO transfection, $5 \times 10^{3}$ cells were incubated with $10 \mu 1$ MTT $(0.5 \mathrm{mg} / \mathrm{ml})$ at $37^{\circ} \mathrm{C}$ for $4 \mathrm{~h}$. The purple formazan crystals were dissolved by the addition of $100 \mu \mathrm{l}$ dimethyl sulfoxide (Tianjin Fu Chen Chemical Reagent Factory, Tianjin, China). The absorbance was measured at a wavelength of $490 \mathrm{~nm}$ using an EAR 340 AT Easy Microplate Reader (SLT Lab-Instruments, SLT Labinstruments GmbH, Salzburg, Austria).

Reverse transcription-quantitative polymerase chain reaction (RT-qPCR). Cellular expression levels of miR-15a, miR-16-1, miR-26a, miR-34a, miR-143 and miR-203 were determined by RT-qPCR (Table I). Total RNA $(1 \mu \mathrm{g})$ from each sample was used to generate complementary (c)DNA with Moloney murine leukemia virus reverse transcriptase (Promega Corporation, Madison, WI, USA). Subsequent to an initial denaturation step at $95^{\circ} \mathrm{C}$ for $10 \mathrm{~min}$ using an Applied Biosystems SYBR Green PCR Master Mix purchased from Thermo Fisher Scientific, Inc., the reaction was subjected to 40 cycles of $95^{\circ} \mathrm{C}$ for $15 \mathrm{sec}$, $60^{\circ} \mathrm{C}$ for $30 \mathrm{sec}$ and $72^{\circ} \mathrm{C}$ for $30 \mathrm{sec}$. An Applied Biosystems 7500 Fast Real-Time PCR Systems (Thermo Fisher Scientific, Inc.) was used to perform cDNA amplification, including routine product checking with the internal dissociation curve software. cDNA transcript quantities were compared using the previously described relative quantification method (19). The amount of detected miRNA was normalized against the amount of the endogenous control, ubiquitin 6. The ratio of the relative value to the control sample was provided as $2^{-\Delta \Delta C q}(19)$.

Terminal deoxynucleotidyl-transferase-mediated deoxynucleotide triphosphate nick end labeling (TUNEL)-staining assays. Subsequent to washing 3 times with phosphate-buffered saline (PBS; Beyotime Institute of Biotechnology, Haimen, China), the cells were fixed with $4 \%$ paraformaldehyde (Beijing Dingguo Changsheng Biotechnology Co., Ltd., Beijing, China) and permeabilized with $0.1 \%$ Triton X-100 (Beijing Solarbio Science \& Technology Co., Ltd., Beijing, China) and $0.1 \mathrm{~mol} / 1$ sodium citrate buffer (Shanghai Haoran Bio Technologies Co., Ltd., Shanghai, China). Subsequently, the In Situ Cell Death Detection kit (Roche Diagnostics, Indianapolis, IN, USA) was used to label apoptotic cells, and the nuclei were stained with 4',6-diamidino-2-phenylindole (Roche Molecular Diagnostics, Pleasanton, CA, USA). The total number of cells and the TUNEL-positive cells were counted using Image-Pro Plus software (Media Cybernetics, Inc., Rockville, MD, USA) to calculate the apoptosis rate, which was defined as the ratio of apoptotic cells to total cells.

Western blot analysis. Total protein was extracted from the cells for protein immunoblotting using a standard protocol. Briefly, $80 \mu \mathrm{g}$ protein samples were separated by $10 \%$ sodium dodecyl sulfate-polyacrylamide gel electrophoresis and blotted onto nitrocellulose membranes. The blots were blocked with $5 \%$ non-fat milk for $2 \mathrm{~h}$ at room temperature and probed with primary antibodies, consisting of rabbit polyclonal anti-PTEN (dilution, 1:1,000; Cell Signaling Technology, Inc., Danvers, MA, USA; cat. no. 9552), rabbit polyclonal anti-p-Akt (dilution, 1:1,000; Cell Signaling Technology, Inc.; cat. no. 9271), 
Table I. Primers used for reverse transcription-quantitative polymerase chain reaction.

Gene name

hsa-miR-15a

hsa-miR-16-1

hsa-miR-26a

hsa-miR-34a

hsa-miR-143

hsa-miR-203

U6
Forward primer, 5'-3'

Reverse primer, 5'-3'

\author{
TAGCAGCACATAATGG \\ TAGCAGCACGTAAATA \\ TTCAAGTAATCCAGGA \\ TGGCAGTGTCTTAGCT \\ TGAGATGAAGCACTG \\ GTGAAATGTTTAGGAC \\ GCTTCGGCAGCACATATACTAAAAT
}

TGCGTGTCGTGGAGTC
TGCGTGTCGTGGAGTC
TGCGTGTCGTGGAGTC
TGCGTGTCGTGGAGTC
TGCGTGTCGTGGAGTC
TGCGTGTCGTGGAGTC
CGCTTCACGAATTTGCGTGTCAT

U6, ubiquitin 6.
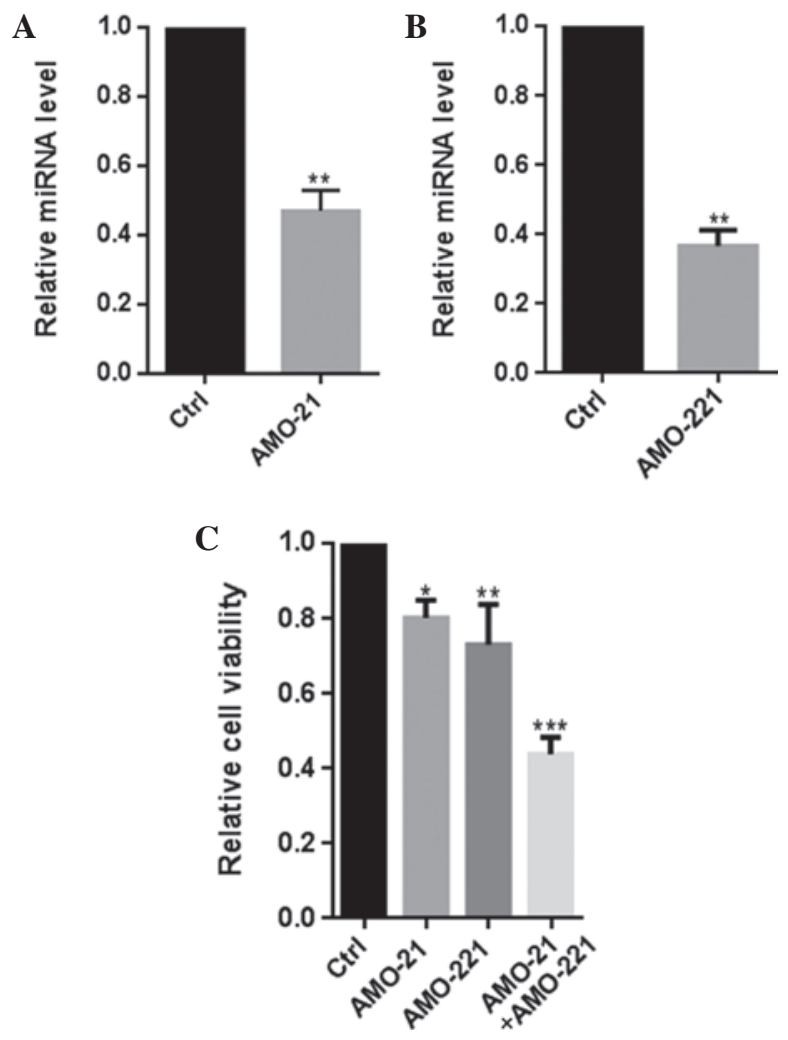

Figure 1. Co-transfection of AMO-21 and AMO-221 decreased the viability of Hep-2 cells $(n=6)$. (A) AMO-21 transfection caused a significant decrease in the expression of miR-21 in Hep-2 cells $\left({ }^{* *} \mathrm{P}<0.01\right.$ vs. control). (B) Transfection with AMO-221 lead to a significant decrease in the expression of miR-221 in Hep- 2 cells $\left({ }^{* *} \mathrm{P}<0.01\right.$ vs. control). (C) The effects of mono-transfections of either AMO-21 or AMO-221 or co-transfection of the two AMOs on cell viability $\left({ }^{*} \mathrm{P}<0.05,{ }^{* *} \mathrm{P}<0.01\right.$ and ${ }^{* * *} \mathrm{P}<0.001$ vs. control. Data are presented as the mean \pm standard error of the mean. AMO, anti-miRNA oligonucleotide; miRNA, microRNA; Ctrl, control.

rabbit polyclonal anti-p53 (dilution, 1:1,000; Cell Signaling Technology, Inc.; cat. no. 2527), rabbit polyclonal anti- $\beta$-actin (dilution, 1:1,000; BD Biosciences, San Jose, CA, USA; cat. no. 612656) antibodies, in PBS and incubated at $4^{\circ} \mathrm{C}$ overnight. Following overnight binding, the membranes were washed with PBS-Triton X-100 and incubated for $1 \mathrm{~h}$ at room temperature with secondary antibodies, as follows: Alexa Fluor ${ }^{\circledR} 700$-conjugated goat anti-mouse IgG (dilution, 1:8,000; Invitrogen; Thermo Fisher Scientific, Inc.; cat. no. A-21036); and IRDye ${ }^{\circledR} 800 \mathrm{CW}$ goat anti-rabbit IgG (dilution, 1:8,000; LI-COR Biosciences, Lincoln, NE, USA; cat. no. 926-32211). Finally, the bands were collected using an Odyssey CLx Infrared Image System fluorescent scanning system (LI-COR Biosciences) and quantified with the Odyssey v1.2 software (LI-COR Biosciences) by measuring intensity of the fluorescence as the area $\mathrm{x}$ optical density. $\beta$-actin was used as an internal control. The results were expressed as fold changes by normalizing the data to the control values.

Statistical analysis. All data were expressed as the mean \pm standard deviation. Statistical analyses, including an unpaired two-tailed Student's t-test, and one-way analysis of variance followed by the Bonferroni's multiple comparison post-hoc test, were conducted using GraphPad Prism 6.0 (GraphPad Software, Inc., La Jolla, CA, USA). P<0.05 was considered to indicate a statistically significant difference.

\section{Results}

Co-transfection of AMO-21 and AMO-221 significantly decreased the cell viability of Hep-2 cells. Previously, it was confirmed that miR-21 and miR-221 were co-overexpressed in patients with LSCC $(7,8)$. In the present study, co-transfection of AMO-21 and AMO-221 were found to clearly reduce the cellular expression of miR-21 and miR-221, leading to a significant decline in the viability of Hep-2 cells, $(\mathrm{P}<0.001$; Fig. 1$)$. Overall, these results indicate a functional interaction between miR-21 and miR-221 in their involvement in the pathology of LSCC.

The results of the MTT assays were verified by observations from the TUNEL experiments. Joint application of AMO-21 and AMO-221 enhanced the promotion of apoptotic alteration in the Hep-2 cells compared with mono-transfection of either AMO at the same concentration (Fig. 2). Co-transfection caused an increase of TUNEL-positive cells between $4 \%$ under normal conditions and almost $50 \%$ post-transfection. Single transfections of AMO-21 or AMO-221 resulted in an increase in TUNEL-positive cells between 4 and $30 \%$. upregulated the expression of PTEN. Low expression of 
A

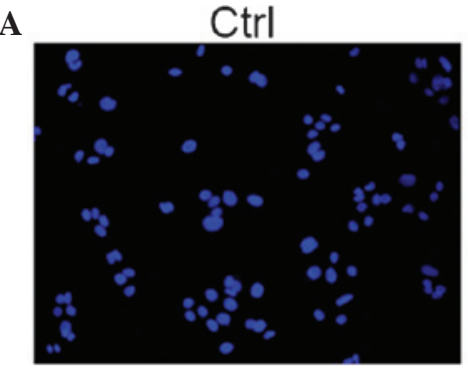

C

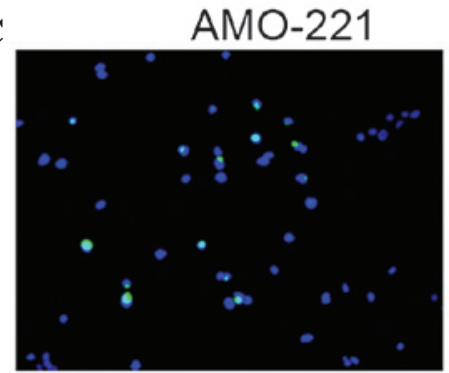

B

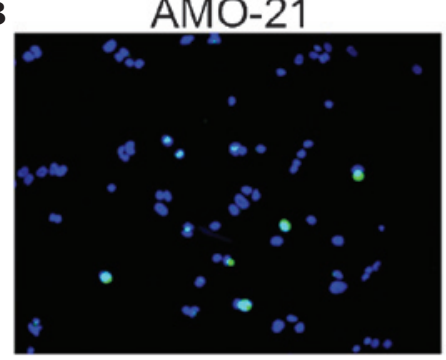

D AMO-21 + AMO-221

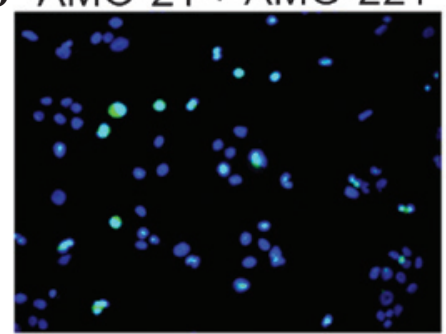

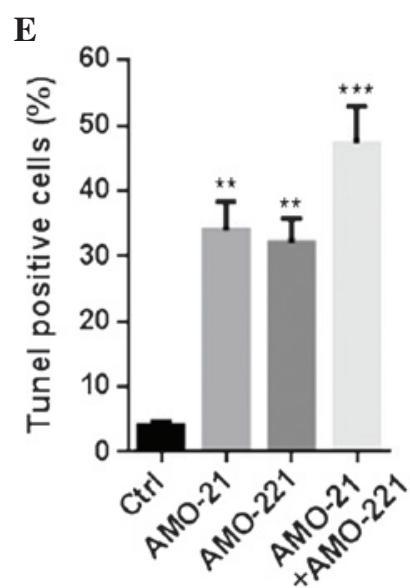

Figure 2. Co-transfection of AMO-21 and AMO-221-induced apoptosis of Hep-2 cells ( $\mathrm{n}=3$ ). Data are presented as the mean \pm standard error of the mean. (A) Normal cells and cells transfected with (B) AMO-21, (C) AMO-221 and (D) AMO-21 and AMO-221. The green staining indicates apoptotic cells and blue staining is the nucleus labeled by 4',6-diamidino-2-phenylindole. (E) Bar chart of TUNEL-positive staining. ${ }^{* *} \mathrm{P}<0.01$ and ${ }^{* * *} \mathrm{P}<0.001$ vs. control. AMO, anti-microRNA oligonucleotide; Ctrl, control; Tunel, terminal deoxynucleotidyl-transferase-mediated deoxynucleotide triphosphate nick end labeling.
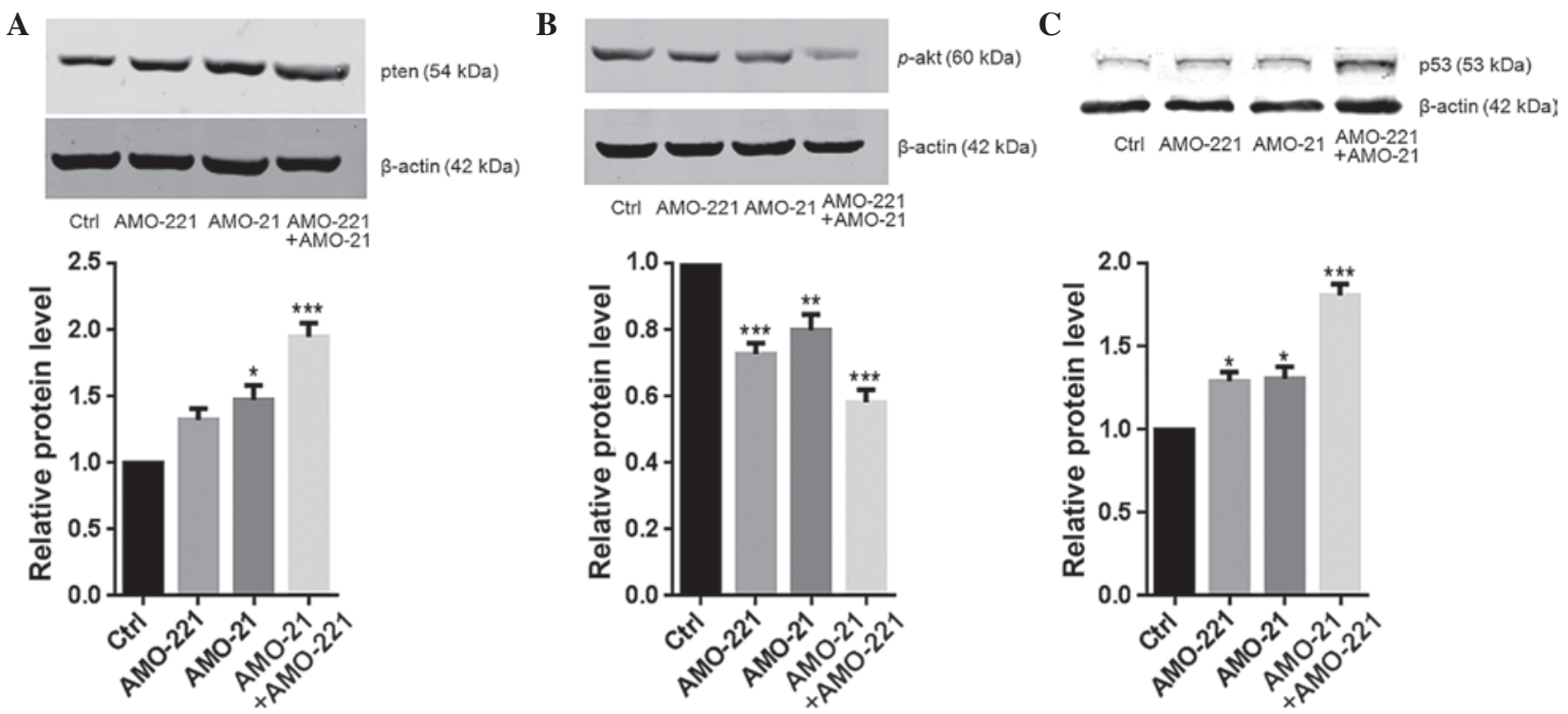

Figure 3. Co-transfection of AMO-21 and AMO-221 inhibited PTEN-Akt signaling and synergistically increased p53 expression in Hep-2 cells. The effects of AMO-21 and AMO-221 on the expression of (A) PTEN, (B) p-Akt and (C) p53 are shown. ${ }^{*} \mathrm{P}<0.05,{ }^{* *} \mathrm{P}<0.01$ and ${ }^{* * * *} \mathrm{P}<0.001$ vs. control; (n=3). Data are presented as the mean \pm standard error of the mean. AMO, anti-microRNA oligonucleotide; Ctrl, control; PTEN, phosphatase and tensin homolog; p-Akt, phosphorylated Akt.

PTEN has been reported in the laryngeal tissue of patients with $\operatorname{LSCC}(11,14)$. The resulting weak negative regulation from PTEN may lead to excessive activation of Akt (14). In the present study, a low cellular content of PTEN and high phosphorylation level of Akt were each observed in the Hep-2 cells (Fig. 3A and B). PTEN is a common target of miR-21 and miR-221 (11,12). The co-transfection of $40 \mathrm{nM}$ of AMO-21 and AMO-221 into Hep-2 cells resulted in an enhanced total cellular concentration of PTEN compared to the untransfected control cells (Fig.3A). Consistently, significant weakening of Akt phosphorylation was observed in the cells that were treated with AMO-21 and AMO-221
$(\mathrm{P}<0.001$; Fig. 3B). As a result, this effect eventually led to an almost 2-fold increase in p53 expression compared with the control group (Fig. 3C). This finding is consistent with a previous study that found Akt negatively controls p53 expression in cancer cells (17).

AMO-21 and AMO-221 synergistically enhanced expression of multiple p53-mediated pro-apoptotic miRNAs. As an important transcription factor, p53 is responsible for the transcription of multiple pro-apoptotic miRNAs, including miR-15a, miR-16-1, miR-26a, miR-34a, miR-143 and miR-203 (18). In the present study, RT-qPCR indicated that 

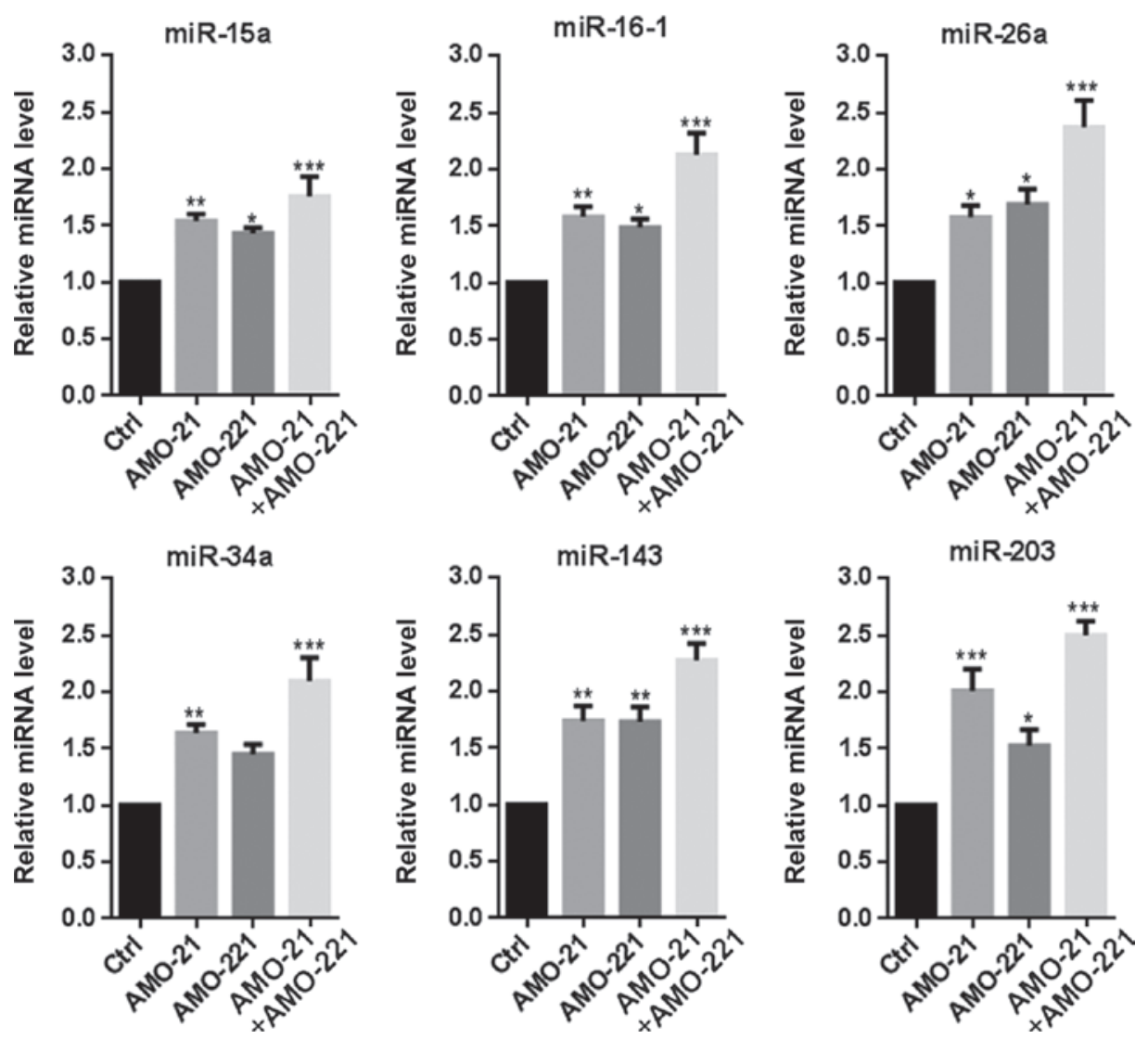

Figure 4. Co-transfection of AMO-21 and AMO-221 resulted in a significant increase in the expression of all 6 pro-apoptotic miRNAs tested, consisting of miR-15a, miR-16-1, miR-26a, miR-34a, miR-143 and miR-203. ${ }^{*} \mathrm{P}<0.05,{ }^{* *} \mathrm{P}<0.01$ and ${ }^{* * *} \mathrm{P}<0.001$ vs. control $(\mathrm{n}=6)$. Data are presented as the mean \pm standard error of the mean. AMO, anti-miRNA oligonucleotide; miRNA, microRNA; Ctrl, control.

the expression of the 6 miRNAs was markedly increased by the concurrent transfection of AMO-21 and AMO-221 in Hep-2 cells (Fig. 4). Enforced transcription of multiple pro-apoptotic miRNAs should be attributed to a significant expression change in p53 (Fig. 3C), suggesting a synergistic effect of AMO-21 and AMO-221 in re-adjusting the balance between anti-apoptotic and pro-apoptotic miRNAs in Hep-2 cells.

\section{Discussion}

miRNAs have broad and important implications in human cancer, which advocates the use of miRNAs as anti-cancer therapeutic targets $(20,21)$. As with numerous other fields of cancer research, miRNA signatures and functions have been persistently revealed in LSCC and associated cancers $(6,7,22)$. Typically, the majority of studies into miRNAs involved in the progression of cancer focus on key individual intervening miRNAs. However, off-target effects due to excessive dosage and non-specific delivery, which is one of the inherent limitations of this approach, should be considered (23). To solve or avoid this problem, an alternative strategy is to combine multiple miRNAs, based on the wide range of synergistic associations that have been suggested in studies investigating miRNAs (24). Co-regulating one target gene or even one biological module may lead to potent synergistic effects by miRNA combinations at a low dose or concentration. The effects shown by miR-10b and miR-21 in glioma and miR-14 and miR-145 in bladder cancer demonstrate the validity of this approach $(25,26)$.

In the present study, the potential synergistic effects of another miRNA pair, miR-21 and miR-221, were investigated in LSCC in vitro. These two miRNAs were each confirmed to be overexpressed in human LSCC tissue (7), target PTEN in the PTEN-Akt signaling pathway $(12,13)$ and share multiple biological modules (10). These findings strongly suggest a possible synergistic interaction between miR-21 and miR-221. Consistent with the reported accumulated data $(10,12,13)$, the present experiments demonstrated synergetic, rather than cumulative, pro-apoptotic effects of co-inhibition with miR-21 and miR-221 in Hep-2 cells. The expression of PTEN was notably increased in the untransfected controls. In addition, a low level of phosphorylation of Akt was observed. It has been well established that PTEN is a pivotal negative regulatory factor of Akt (13), whereas excessive activation of Akt was found to be strongly associated with LSCC metastasis (14). Overactivation of Akt ultimately leads to an increase in the expression of the tumor suppressor gene TP53 (15). Briefly, decreased intensity of PTEN-Akt signaling reduced the inhibitory role of PTEN-Akt signaling on this downstream p53 pathway. Thus, it was not notable that in the present study a clear increase in the expression of p53 was found by blocking the miRNA-mediated regulation of PTEN with co-transfection of AMO-21 and AMO-221.

Furthermore, the present findings suggest that p53 may play a central role in the balance of the function of anti-apoptotic 
and pro-apoptotic miRNAs in Hep-2 cells. Downregulation of anti-apoptotic or tumor-promoting miRNAs, such as miR-21 and miR-221, led to the upregulation of the 6 pro-apoptotic miRNAs tested in the present study, consisting of miR-15a, miR-16-1, miR-26a, miR-34a, miR-143 and miR-203. This may also result in the phenomenon of co-inhibition with miR-21 and miR-221 results in reduced cell viability and apoptosis in Hep-2 cells, observed by MTT and TUNEL-staining assays. By co-regulating the target gene PTEN and affecting the PTEN-Akt and p53 signaling pathways, miR-21 and miR-221 exert functional control on the cellular expression of these proteins with opposing functions and promote cancer cell proliferation. By contrast, this specific mechanism of a miRNA mutual interaction highlights the unique advantage of the combination of AMO-21 and AMO-221 as a potential treatment of LSCC, compared with the traditional strategy of overexpressing a single pro-apoptotic miRNA or downregulating a single tumor-promoting miRNA (22). Additionally, the joint application of two AMOs, such as AMO-21 and AMO-221, overcomes the potential issue of the non-specific effects of miRNA, because only low concentrations or doses of the AMOs are required.

In conclusion, to the best of our knowledge, the present in vitro study assessed for the first time the potential synergistic pro-apoptotic effects of miR-21 and miR-221 in the LSCC Hep-2 cell line, by co-inhibition of the two tumor-promoting miRNAs at a low intervention level. Novel insights were also obtained for the mechanism underlying miRNA-miRNA interactions in balancing and adjusting the cellular expression and function of each interacting partner. The present findings indicate that the combination of AMO-21 and AMO-221 should be considered to possess therapeutic potential for future LSCC treatments. However, additional in vivo functional experiments are required to validate the present findings.

\section{Acknowledgements}

The present study was supported by grants from the Foundation of Heilongjiang Provincial Health and Family Planning Commission (grant no. 2014-309), the Talented Youth Fund of Harbin Municipal Science and Technology Bureau (grant no. 2015RAQYJ076), the Postdoctoral Fund (grant nos. LBH-Z12157 and 2014M551276), and the National Science Foundation of China (grant nos. 81241085, 81372902 and 81402234).

\section{References}

1. Genden EM, Ferlito A, Silver CE, Jacobson AS, Werner JA, Suárez C, Leemans CR, Bradley PJ and Rinaldo A: Evolution of the management of laryngeal cancer. Oral Oncol 43: 431-439, 2007.

2. Marioni G, Marchese-Ragona R, Cartei G, Marchesex F and Staffieri A: Current opinion in diagnosis and treatment of laryngeal carcinoma. Cancer Treat Rev 32: 504-515, 2006.

3. Zheng Y, Liu M, Li M, Zhang J, Ge J, Sun Y and Tian L: The influence of the 'patient-to-patient model' on swallowing problems in patients with supraglottic laryngeal cancer. ORL J Otorhinolaryngol Relat Spec 76: 171-177, 2014.

4. Jenckel F and Knecht R: State of the art in the treatment of laryngeal cancer. Anticancer Res 33: 4701-4710, 2013
5. Ma J, Liu Y, Huang XL, Zhang ZY, Myers JN, Neskey DM and Zhong LP: Induction chemotherapy decreases the rate of distant metastasis in patients with head and neck squamous cell carcinoma but does not improve survival or locoregional control: A meta-analysis. Oral Oncol 48: 1076-1084, 2012.

6. Nohata N, Hanazawa T, Kinoshita T, Okamoto Y and Seki N: MicroRNAs function as tumor suppressors or oncogenes: Aberrant expression of microRNAs in head and neck squamous cell carcinoma. Auris Nasus Larynx 40: 143-149, 2013.

7. Cao P, Zhou L, Zhang J, Zheng F, Wang H, Ma D and Tian J: Comprehensive expression profiling of microRNAs in laryngeal squamous cell carcinoma. Head Neck 35: 720-728, 2013.

8. Ren J, Zhu D, Liu M, Sun Y and Tian L: Downregulation of miR-21 modulates Ras expression to promote apoptosis and suppress invasion of Laryngeal squamous cell carcinoma. Eur J Cancer 46: 3409-3416, 2010.

9. Sun X, Liu B, Zhao XD, Wang LY and Ji WY: MicroRNA-221 accelerates the proliferation of laryngeal cancer cell line Hep-2 by suppressing Apaf-1. Oncol Rep 33: 1221-1226, 2015.

10. Zhu W, Zhao Y, Xu Y, Sun Y, Wang Z, Yuan W and Du Z: Dissection of protein interactomics highlights microRNA synergy. PLoS One 8: e63342, 2013.

11. Li D, Feng J, Wu T, Wang Y, Sun Y, Ren J and Liu M: Long intergenic noncoding RNA HOTAIR is overexpressed and regulates PTEN methylation in laryngeal squamous cell carcinoma. Am J Pathol 182: 64-70, 2013.

12. Meng F, Henson R, Wehbe-Janek H, Ghoshal K, Jacob ST and Patel T: MicroRNA-21 regulates expression of the PTEN tumor suppressor gene in human hepatocellular cancer. Gastroenterology 133: 647-658, 2007.

13. Garofalo M, Di Leva G, Romano G, Nuovo G, Suh SS, Ngankeu A, Taccioli C, Pichiorri F, Alder H, Secchiero P, et al: miR-221\&222 regulate TRAIL resistance and enhance tumorigenicity through PTEN and TIMP3 downregulation. Cancer Cell 16: 498-509, 2009.

14. Li R, Wang R, Zhai R and Dong Z: Targeted inhibition of mammalian target of rapamycin (mTOR) signaling pathway inhibits proliferation and induces apoptosis of laryngeal carcinoma cells in vitro. Tumori 97: 781-786, 2011.

15. Paez J and Sellers WR: PI3K/PTEN/Akt pathway. A critical mediator of oncogenic signaling. Cancer Treat Res 115: 145-167, 2003.

16. Yu C, Liu Y, Tan H, Li G, Su Z, Ren S, Zhu G, Tian Y, Qiu Y and Zhang X: Metadherin regulates metastasis of squamous cell carcinoma of the head and neck via Akt signalling pathway-mediated epithelial-mesenchymal transition. Cancer Lett 343: 258-267, 2014.

17. Abraham AG and O'Neill E: PI3K/Akt-mediated regulation of p53 in cancer. Biochem Soc Trans 42: 798-803, 2014.

18. Suzuki HI, Yamagata K, Sugimoto K, Iwamoto T, Kato S and Miyazono K: Modulation of microRNA processing by $\mathrm{p} 53$. Nature 460: 529-533, 2009.

19. Livak KJ and Schmittgen TD: Analysis of relative gene expression data using real-time quantitative PCR and the $2^{-\Delta \Delta C T}$ method. Methods 25: 402-408, 2001.

20. Garzon R, Calin GA and Croce CM: MicroRNAs in Cancer. Annu Rev Med 60: 167-179, 2009.

21. Garzon R, Marcucci G and Croce CM: Targeting microRNAs in cancer: Rationale, strategies and challenges. Nat Rev Drug Discov 9: 775-789, 2010.

22. Sethi N, Wright A, Wood H and Rabbitts P: MicroRNAs and head and neck cancer: Reviewing the first decade of research. Eur J Cancer 50: 2619-2635, 2014.

23. Singh S, Narang AS and Mahato RI: Subcellular fate and off-target effects of siRNA, shRNA and miRNA. Pharm Res 28: 2996-3015, 2011.

24. Xu J, Li CX, Li YS, Lv JY, Ma Y, Shao TT, Xu LD, Wang YY, Du L, Zhang YP, et al: MiRNA-miRNA synergistic network: Construction via co-regulating functional modules and disease miRNA topological features. Nucleic Acids Res 39: 825-836, 2011.

25. Dong CG, Wu WK, Feng SY, Wang XJ, Shao JF and Qiao J: Co-inhibition of microRNA-10b and microRNA-21 exerts synergistic inhibition on the proliferation and invasion of human glioma cells. Int J Oncol 41: 1005-1012, 2012.

26. Noguchi S, Yasui Y, Iwasaki J, Kumazaki M, Yamada N, Naito S and Akao Y: Replacement treatment with microRNA-143 and -145 induces synergistic inhibition of the growth of human bladder cancer cells by regulating PI3K/Akt and MAPK signaling pathways. Cancer Lett 328: 353-361, 2013. 